\title{
Cycle biogéochimique de l'aluminium. Influence d'une substitution d'espèce : remplacement du chêne (Quercus sessiliflora) par de l'épicéa commun (Picea abies)
}

\author{
C. NYS \\ avec la collaboration technique de Louisette GELHAYE et D. GELHAYE \\ INRA, Station de Recherches sur le Sol, la Microbiologie et la Nutrition des Arbres forestiers, \\ Centre de Recherches de Nancy, Champenoux, F 54280 Seichamps
}

\begin{abstract}
Summary
The biogeochemical cycle of aluminium : the effect of replacing oak forest (Quercus sessiliflora) by Norway spruce (Picea abies)
\end{abstract}

The substitution of oak forest by Norway spruce induces changes in the physico-chemical properties of soil and in the dynamics of the biogeochemical cycle of clements. Aluminium is a good indicator of pedogenesis. Do conifers induce podzolisation in an acid brown earth soil (« sol brun acide ")?

In the French Ardennes, on a brown soil, after the substitution of tree species, the mull humus under the oak forest became a moder after 50 years of spruce cultivation. The consequence of the change on the soil chemical properties is an increase in exchangeable acidity (fig. 2) in the litter and the upper organic layer of the soil $\left(A_{1}\right)$. In relation to the increase of organic matter, the C.E.C. and the Al concentration are greater under spruce (fig. 3 ) in the $A_{1}$ level; but the acidity ratio $A l / C$.E.C. remains the same.

In figure 4 , the weathering complex shows higher levels of translocation of free Al under spruce, with adsorption in the (B) horizon. That is typical under the deciduous forest of a sol brun acide", and under the spruce forest of a « sol brun ocreux ».

The evolution of the soil towards the podzolic group due to conifer plantation has been verified by a study of flux and in particular, the $\mathrm{Al}$ flux in the ecosystems.

Aluminium flux has been evaluated in the two ecosystems using lysimeter techniques.

A comparison of Al distribution in the two ecosystems shows that no difference exists in the upper part of ecosystems (similar biological cycles) : current immobilization in biomass and flux by litterfall or throughfall are the same. The input of Al by wet or dry deposits are negligible.

The differences in the processes are greater in the soil :

i) concentration (fig. 6 top): the species effect is greater than the seasonal effect, with an increase in concentration in the $A_{1}$ level and relatively stable concentrations in the (B) and (B) C levels. The difference in concentration between the two species is due to the difference in the $A_{1}$ level.

ii) flux (fig. 6, bottom) : because of lower drainage levels under the coniferous forest the shapes of the curves are different. The seasonal effect is significant for the two soils and is due to the water flux. The species effect is less obvious, but it shows a differentiation between processes. $A l$ is released in the $A_{1}$ level and translocated below the $C$ level. The principal difference under the spruce stand is the adsorption of a part of this aluminium in the $B$ (C) level.

The values of the correlation coefficient show a good relationship between $A l$ and $C$ in the $A_{1}$ level under spruce (chelate), and $\mathrm{AJ}$ and $\mathrm{N}-\mathrm{NO}_{3}$ and $\mathrm{S}-\mathrm{SO} 4$ in the (B) C levels in the two 
ecosystems. but in this study we have not determined the presence or absence of a complexed form of Al to demonstrate that the difference is due to an evolution of the processes from acidification towards podzolization. The comparative Al balance for the two ecosystems shows (fig. 7) a similarity between the input and output in the two ecosystems, but shows a differentiation within the soil, with the adsorption of about $5 \mathrm{~kg} \cdot \mathrm{ha}^{-1} \cdot \mathrm{y}^{-1}$ of aluminium in the (B)C level which had been released from the $A_{1}$ level.

Key words: Aluminium, biogeochemical cycle, forest, deciduous coniferous, spruce.

\section{Résumé}

L'introduction de lépicéa commun en remplacement dun taillis sous futaie de chênes provoque des modifications de l'humus du mull au moder après 50 ans de monoculture.

Les changements de caractéristiques du sol sont nets pour laluminium. Il y a augmentation de la capacité d'échange sous les résineux et de l'aluminium échangeable. Le profil de redistribution de l'alumine libre (C.B.D.) montre une migration de cette forme d’aluminium sous les résineux. Les indices de redistribution de Souciner (1971) ont pour valeur 1,4 sous les feuillus et 2,0 sous les épicéas, indice caractérisant une podzolisation commençante sous le résincux.

Les études des flux par des systèmes lysimétriques sans tension permettent de comparer le fonctionnement actuel des deux écosystèmes. Ces deux écosystèmes se différencient déjà par les flux d'eau gravitaire. en effet l'interception des pluies incidentes par les peuplements est plus élevée sous l'épicéa $(30$ p. 100$)$ que sous les feuillus $(10$ p. 100$)$.

Les prolils moyens annuels ou saisonniers montrent qu'il n'y a pas d'apport extérieur par les pluies incidentes et que le cycle biologique a un rôle peu important dans les restitutions sous les feuillus, mais relativement plus pour lépicéa.

Les flux daaluminium augmentent sous les deux peuplements sous lhorizon $A_{1}$ signe d'une altération des minéraux primaires. Cet aluminium est faiblement redistribué dans l'horizon (B) sous les feuillus et fortement dans Thorizon (B) et B (C) sous les résineux, ce qui caractérise un effet hautement significatif de l'espèce. L'effet de la satison est significatif, il joue par l'effet du flux d'eau ou effet de dilution. Sous les épicéas l'aluminium migre essenticllement en automne et en hiver (84 p. 100).

Le calcul des corrélations entre éléments montre qu'en surface, dans l'horizon $A_{1}$, l'aluminium et le carbone sont liés entre cux mais quau niveau des horizons (B) C du sol, les coefficients de corrélation sont hautement significatifs entre aluminium et nitrate ainsi quentre aluminium et sulfate laissant à penser qu une partie de laluminium migre hors du sol en liaison avec ces deux anions.

L'épicéa commun provoque des modifications des caractéristiques du sol et de son fonctionnement. L'action podzolisante de cette espèce, sur un sol brun acide contenant $10 \mathrm{p}$. 100 d'argile. est faible mais significative et caractérise un changement dans le cycle biogéochimique de l'aluminium.

Mots clés: Aluminium, cycle biogéochimique, forêt, feuillus, résineux, épicéa.

\section{Introduction}

La mise en valeur des forêts feuillues peu productives par des plantations résineuses a pour conséquence des modifications physico-chimiques et biologiques (Nys. 1987). Le point de départ des modifications édaphiques est l'accumulation de matière organique sous les conifères (Delecour et al., 1967 ; NolRfai.ise \& VANESSE, 1975). Le cycle biologique est perturbé et les composés organiques acides ou complexants nés dans les horizons $A_{0}$ ne sont pas dégradés en $A_{1}$ et peuvent agresser les minéraux du 
sol (D.G.R.S.T., 1977). Les composés organiques libérés par l'épicéa commun vont-ils accélérer l'altération des minéraux primaires et vont-ils entraîner un profond changement dans le fonctionnement du sol. Il y aura-t-il même amorce du processus de podzolisation?

Cet article rend compte des transformations concernant l'aluminium, élément indicateur de la pédogénèse, dans le sol et au cours de son cycle biogéochimique dans l'écosystème, sous un peuplement d'épicéas d'une cinquantaine d'années, comparativement à un peuplement feuillu mitoyen.

\section{Matériels et méthodes}

\subsection{Le site}

La station étudiée est située en forêt de Château-Regnault dans les Ardennes. L'altitude est d'environ $390 \mathrm{~m}$ et la pente de 4 à 6 pour cent. La pluviométrie moyenne annuelle, de 1978 à 1983 , a été de $1300 \mathrm{~mm}$ et la température moyenne annuelle est de $8{ }^{\circ} \mathrm{C}$.

Le sol sous le peuplement feuillu est un sol brun acide à mull développé sur un schiste Révinien recouvert de limon d'origine locale. Les caractéristiques chimiques sont rassemblées dans le tableau 1 . Sous le peuplement résineux, le sol est maintenant un sol brun ocreux. L'humus est de type moder (NYS, 1981).

Le peuplement feuillu est un taillis sous-futaie à réserves de chênes (Quercus sessiliflora Salisb.) et de quelques hêtres (Fagus sylvatica L.). Le taillis âgé de 31 ans (en 1981) est composé de sorbier (Sorbus aucuparia L.), de bouleau (Betula verrucosa E.) et d'espèces diverses (Quercus sessiflora, Corilus avellana).

Le peuplement résineux est une plantation d'Epicéa commun (Picea abies L. Karst) âgée de 50 ans (en 1981).

\subsection{Les stocks dans le sol et les immobilisations dans la biomasse}

\subsection{Le sol}

Les évaluations des stocks d'éléments dans le sol ont été réalisées à partir du prélèvement de 10 horizons de 5 profils de sol dans chacun des deux peuplements. Le calcul isoquartz (Nys, 1981) a permis d'évaluer le foisonnement dû à l'espèce, et de choisir des épaisseurs équivalentes de profils.

L'aluminium échangeable a été extrait par une solution de $\mathrm{KCl}$ normale, l'aluminium libre par le réactif C.B.D. (Citrate, Bicarbonate, Dithionite de sodium) et la forme totale a été déterminée par fusion au métaborate de strontium. Les analyses ont été effectuées par absorption atomique.

\subsection{Immobilisation dans la biomasse}

Les résultats des évaluations de la biomasse sont décrits par Nys et RanGER (1981). Les immobilisations d'aluminium ont été calculées à partir de ces chiffres de biomasse et d'analyses de laluminium dans les différents compartiments des arbres. 


\subsection{Flux dans l'écosystème}

\subsection{Sous la forme solide}

\section{- Accroissement annuel dans la biomasse}

L'accroissement annuel dans la biomasse est déterminé par différence à partir du modèle de biomasse établi pendant l'année $\mathrm{n}$ et appliqué aux inventaires des peuplements pour l'année $n+1$.

- Restitution par la litière et la strate herbacée

Les chutes de litières ont été collectées mensuellement dans des bacs $(280 \times 545 \mathrm{~mm})$ situés sous la cime des peuplements. La strate herbacée est collectée sur 30 emplacements de surface $0,1 \mathrm{~m}^{2}$. L'accroissement du stock d'aluminium immobilisé dans les arbres morts dans l'année a été évalué par un modèle de régression.

\section{- Dépôts secs}

Ils ont été évalués selon le principe de Miller (1984). Ils concernent essentiellement les apports externes de soufre et d'azote (Nys, 1987).

\subsection{Sous la forme liquide}

Les eaux de gravité circulant dans les deux écosystèmes ont été collectées par des dispositifs de lysimétrie (fig. 1) qui sont :

- précipitations incidentes dans des pluviomètres de types météorologie nationale situés dans des zones de plaines ouvertes distantes de $100 \mathrm{~m}$ à moins de $5 \mathrm{~km}$ des peuplements :

- pluviolessivats et écoulement le long du tronc: 5 pluviomètres $(280 \times 545 \mathrm{~mm})$ ont été placés systématiquement à $5 \mathrm{~m}$ de distance les uns des autres sous les cimes des peuplements. L'écoulement le long du tronc a été estimé selon Aussenac (1975).

\section{- Composition des eaux de gravité}

4 plaques lysimétriques sans tension ont été installées pour collecter les eaux de gravité dans le sol à chacun des niveaux suivants: litière, horizon $A_{1}(-10 \mathrm{~cm})$, horizon (B) $(-30 \mathrm{~cm})$ et horizon $(B) C(-60 \mathrm{~cm})$.

Ce système respecte les paramètres du cycle biologique (prélèvement par le peuplement) mais ne permet pas l'évaluation des flux d'éléments. Les eaux sont prélevées mensuellement, l'aluminium est analysé après filtration par absorption atomique.

\section{- Evaluation du drainage}

Le drainage a été estimé pour chacun des niveaux à partir des relevés climatologiques (Nys, 1987) et calculé selon l'équation suivante:

$$
\mathrm{D}=\mathrm{Pi}-\mathrm{I}-\mathrm{ETR}-\Delta \mathrm{W}
$$

$\mathrm{D}=$ drainage

$\mathrm{Pi}=$ précipitation incidente

ETR = évapotranspiration des peuplements calculée selon TuRC (1961)

$I$ = interception par les peuplements

$\Delta \mathrm{W}=$ variations des réserves en eau du sol 


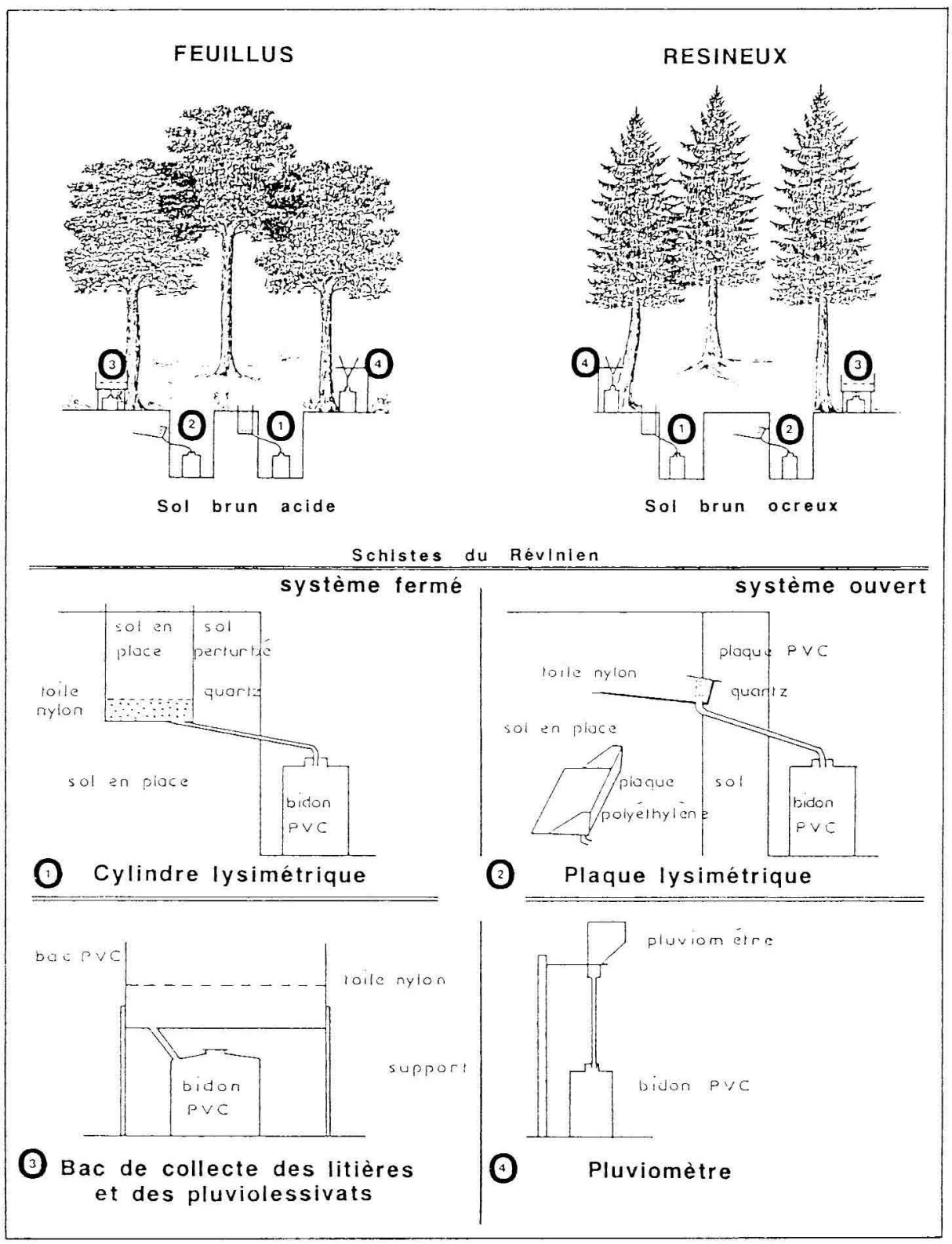

FIG. 1

Schéma des installations de mesure des flux d'éléments dans la station expérimentale de Monthermé (Ardennes).

Diagramme of measurement equipment for element flux at the Monthermé (Ardennes) experimental site. 


\section{Résultats}

\subsection{L'aluminium dans les sols}

\subsection{Le complexe d'échange}

Les acidités libre et d'échange sont plus élevées en surface dans l'horizon $A_{1}$ sous les épicéas (fig. 2). La moyenne générale de la capacité d'échange cationique (C.E.C.) est à un niveau général faible : $6,1 \mathrm{meq} / 100 \mathrm{~g}$. Elle décroît rapidement avec la profondeur (tabl. 1). L'effet espèce n'est marqué que dans les tout premiers centimètres du profil, au niveau de l'horizon $A_{n}$. Cet accroissement de C.E.C. est lié essentiellement à celui de la matière organique.

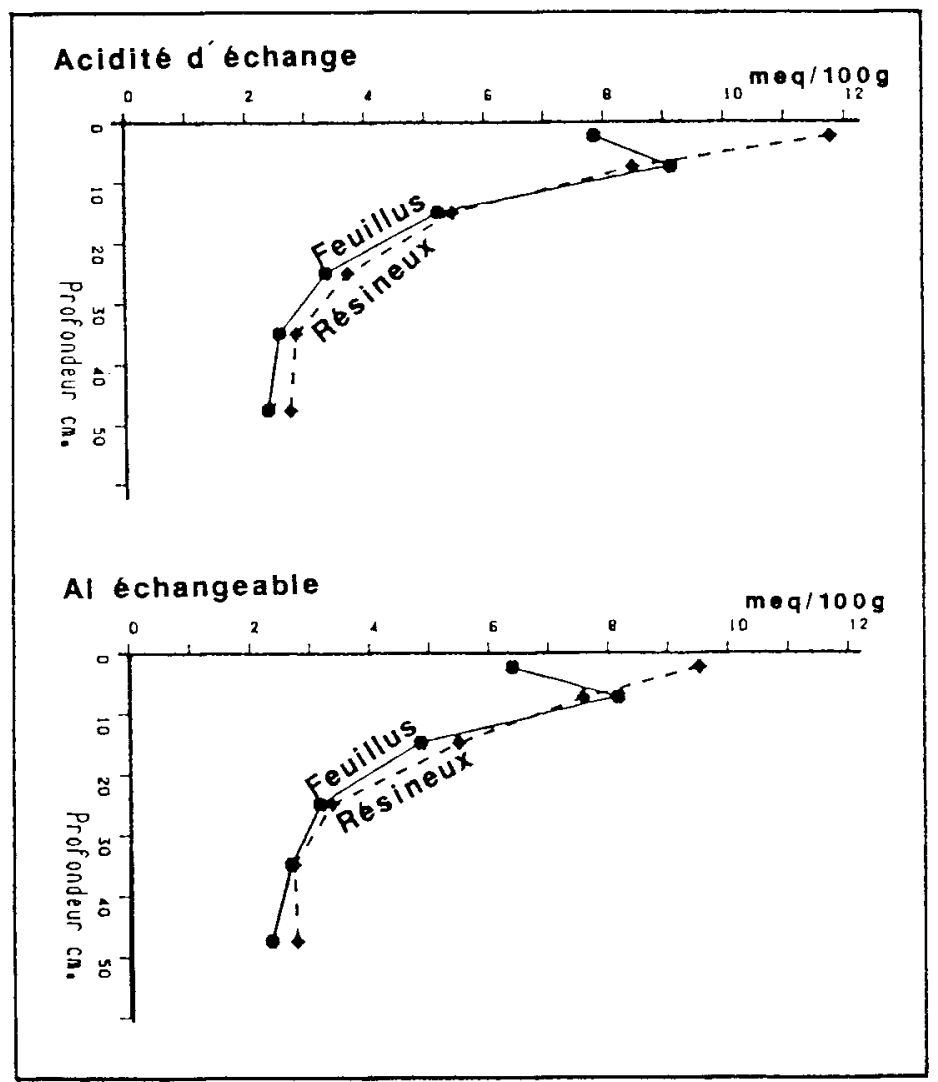

FIG. 2

Courbes de répartition de l'acidité d'échange et de l'aluminium échangeable dans les profils de sols sous feuillus et résineux.

Distribution curves for exchangeable acidity and aluminium in the soil profiles under deciduous and coniferous stands. 


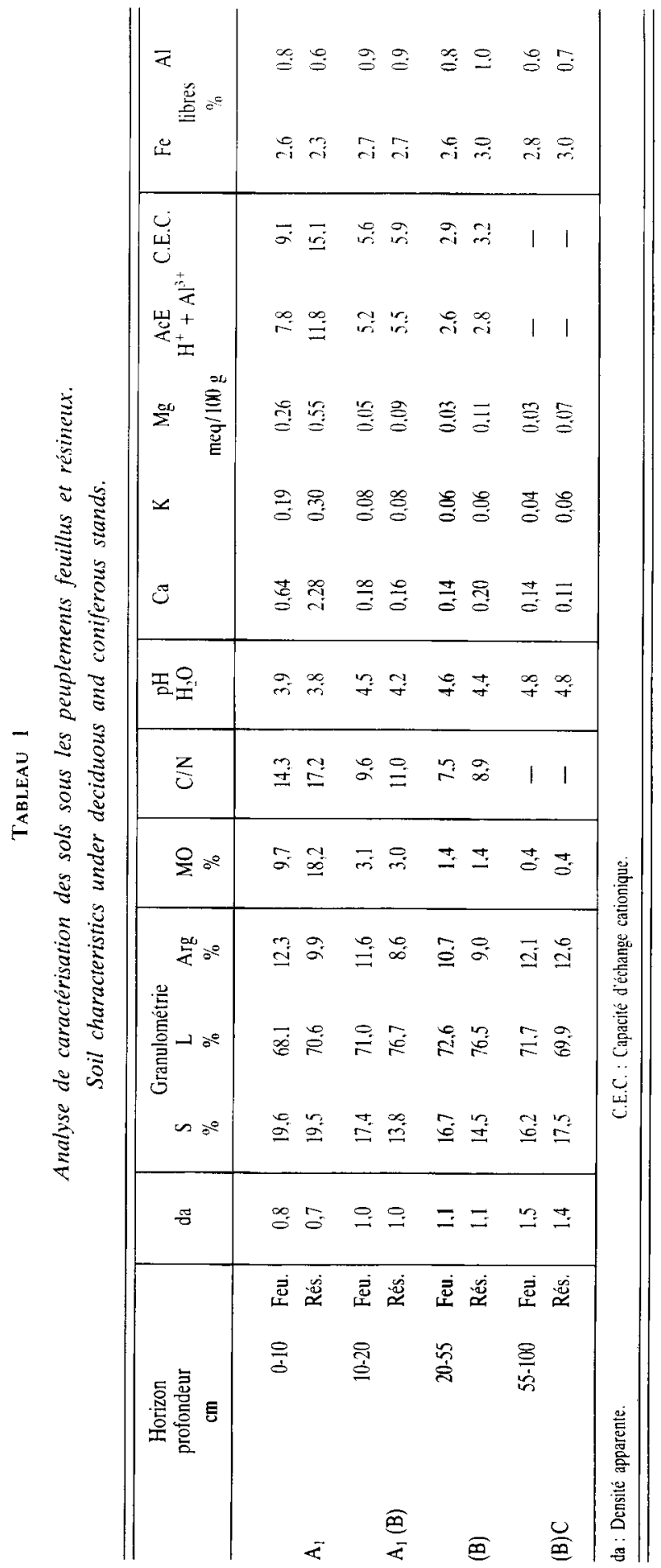




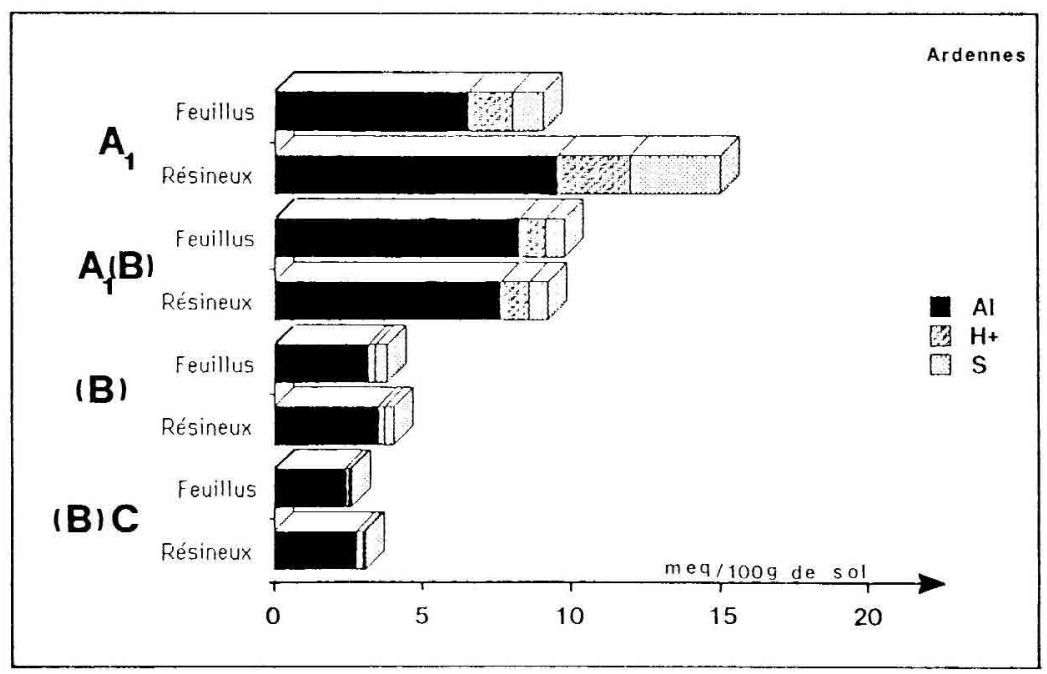

FIG. 3

Modification de la capacité d'échange cationique et de l'occupation de ses sites d'échanges pour les sols des deux peuplements.

Modification of the cation exchange capacity and the composition of the elements on the exchange sites in the soils of the two stands.

Dans ces sols acid̉es, ph variant de 3,8 à 4,8 , la saturation est due principalement aux cations à caractère acide, en particulier l'aluminium. La figure 3 montre que l'aluminium échangeable occupe la majeure partie des sites d'échanges. La teneur en aluminium, exprimée en meq $/ 100 \mathrm{~g}$ de sol, est plus élevée sous les résineux que sous les feuillus. Par contre le taux d'acidité (Espinu \& Pedro, 1980) est plus faible caractérisant l'augmentation des cations basiques sous les épicéas.

\subsection{Le complexe d'altération}

Que ce soit en concentration ou en variation isoquartz des quantités, l'aluminium libre montre sur la figure 4 des distributions différentes entre les espèces feuillues et résineuses. Sous l'Epicéa cette forme d'aluminium migre et s'accumule plus profondément que sous les feuillus.

Les coefficients de redistribution $\mathrm{K}_{\mathrm{Al}}$ et $\mathrm{K}_{\mathrm{Fe}}$ de Souchier (1971) montrent que ceuxci sont plus élevés pour les sols sous résineux que ceux sous feuillus :

$$
\begin{array}{ll}
\mathrm{K}_{\mathrm{Al}} \text { (résineux) }=2,0 & \mathrm{~K}_{\mathrm{Al}} \text { (feuillus) }=1,4 \\
\mathrm{~K}_{\mathrm{Fe}} \text { (résineux) }=1,15 & \mathrm{~K}_{\mathrm{Fc}} \text { (feuillus) }=1,0
\end{array}
$$

Par comparaison avec ceux cités par cet auteur, les valeurs de ces indices sont caractéristiques pour les feuillus des sols bruns acides et pour les résineux des sols cryptopodzoliques ou bruns ocreux.

\subsection{Les éléments totaux} résineux.

Il n'y a pas de différence significative entre les profils sous feuillus et sous les 


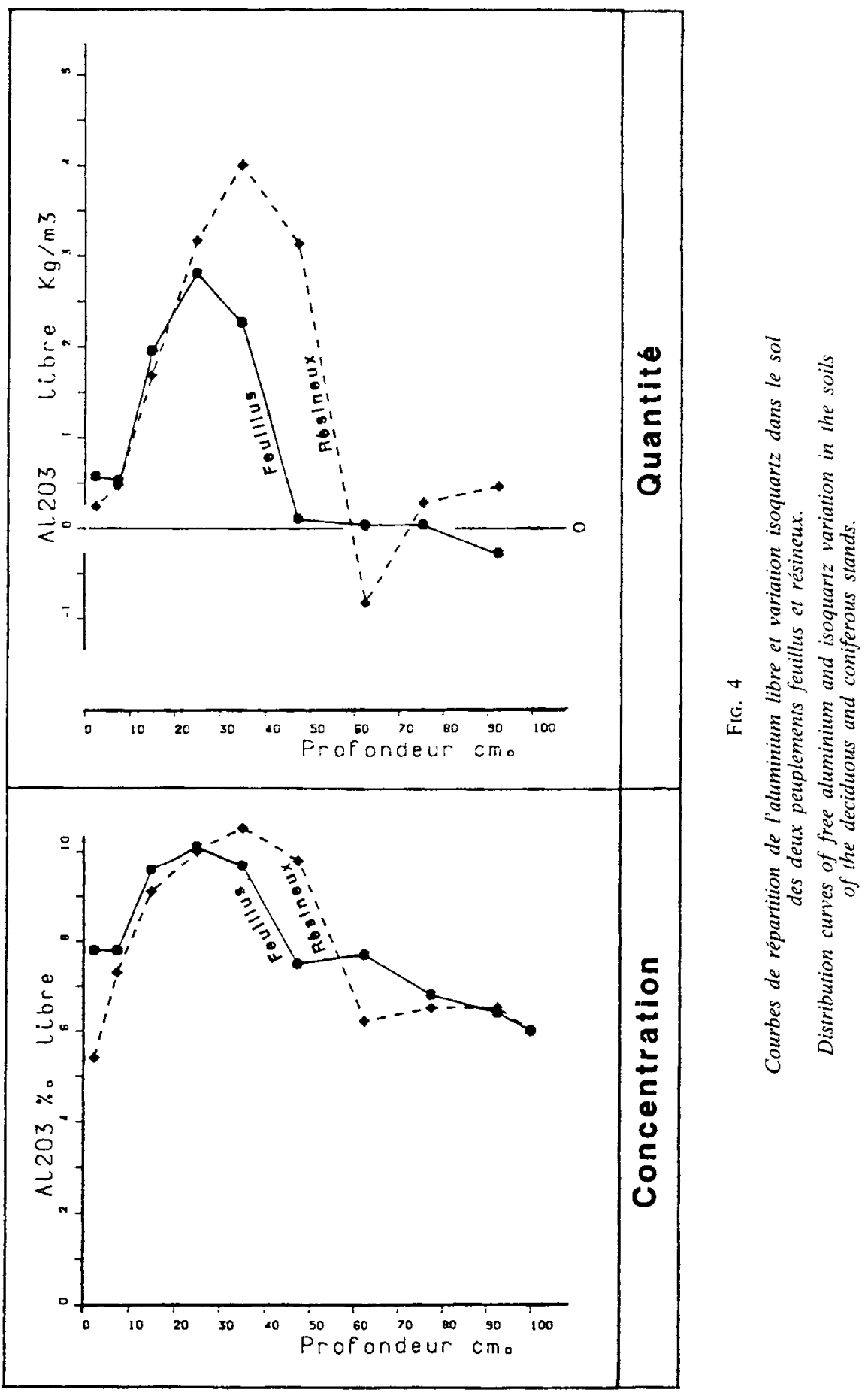



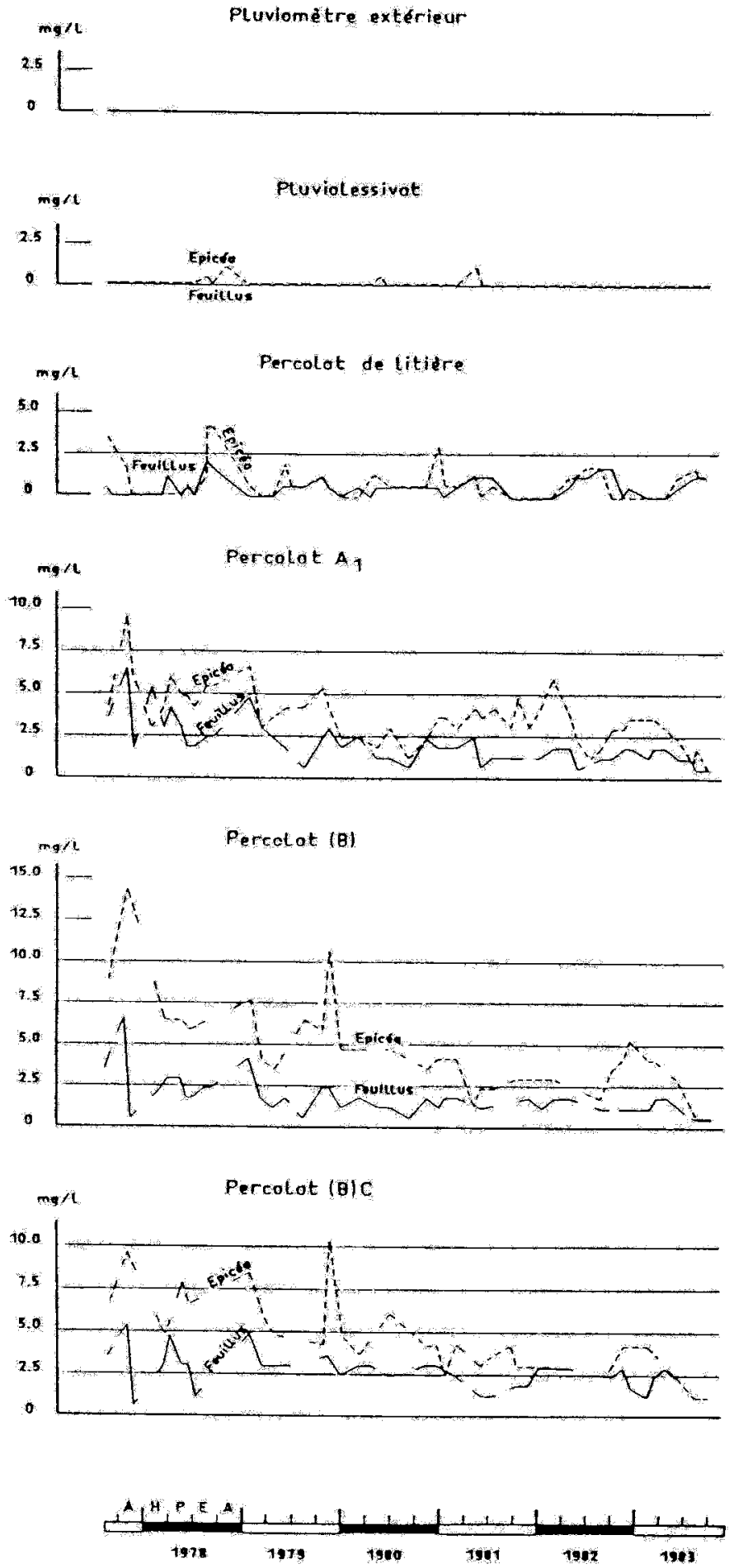


\section{Acidité à $\mathrm{pH} \mathbf{8 . 4}$}
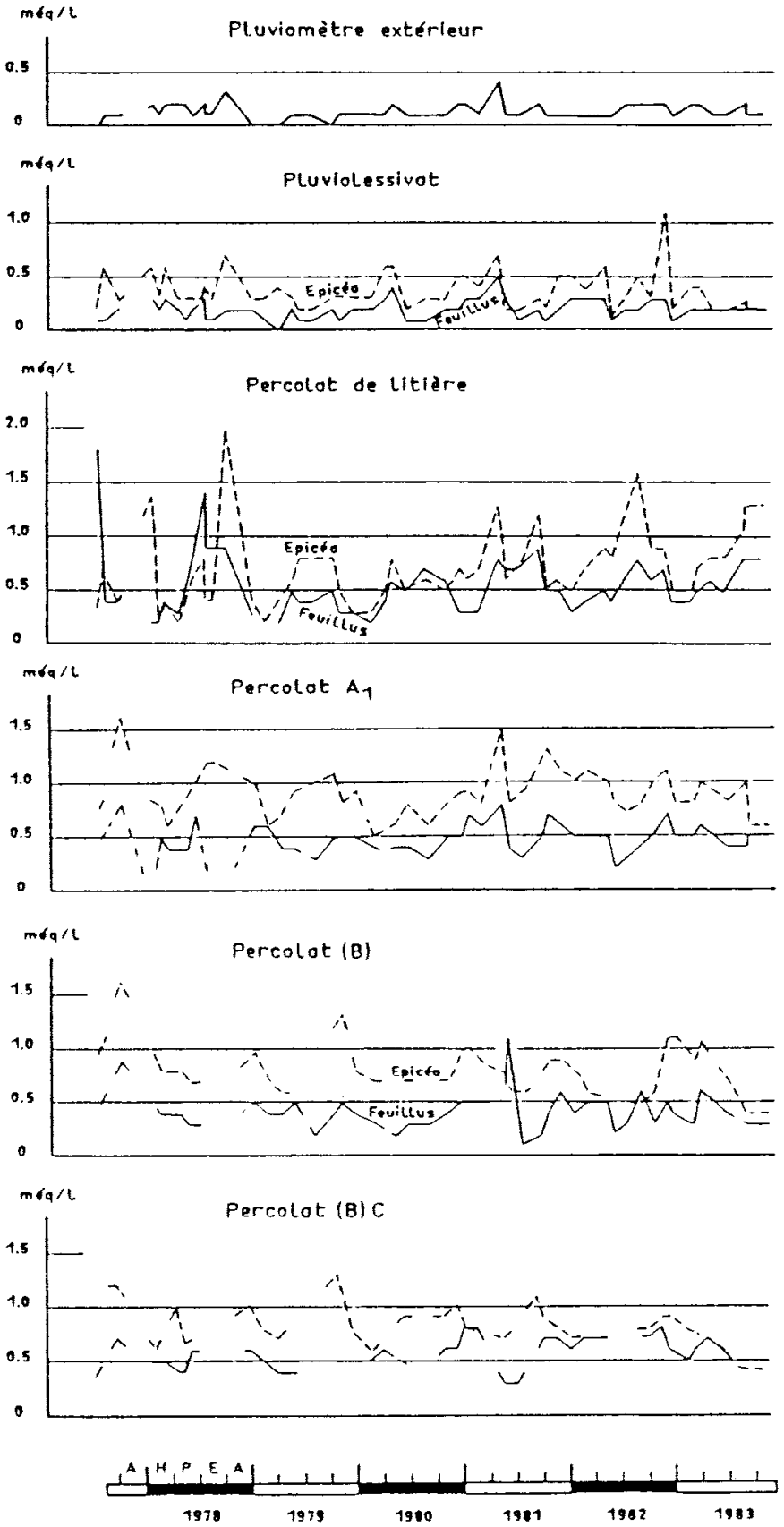

Fig. 5

Evolutions saisonnières des concentrations en aluminium et de l'acidité à pH 8.4 aux différents niveaux de prélèvement dans les deux peuplements feuillus et résineux.

Seasonal variations of aluminium concentration and acidity at $\mathrm{pH} 8.4$ for the different sampling levels in the deciduous and coniferous stands. 


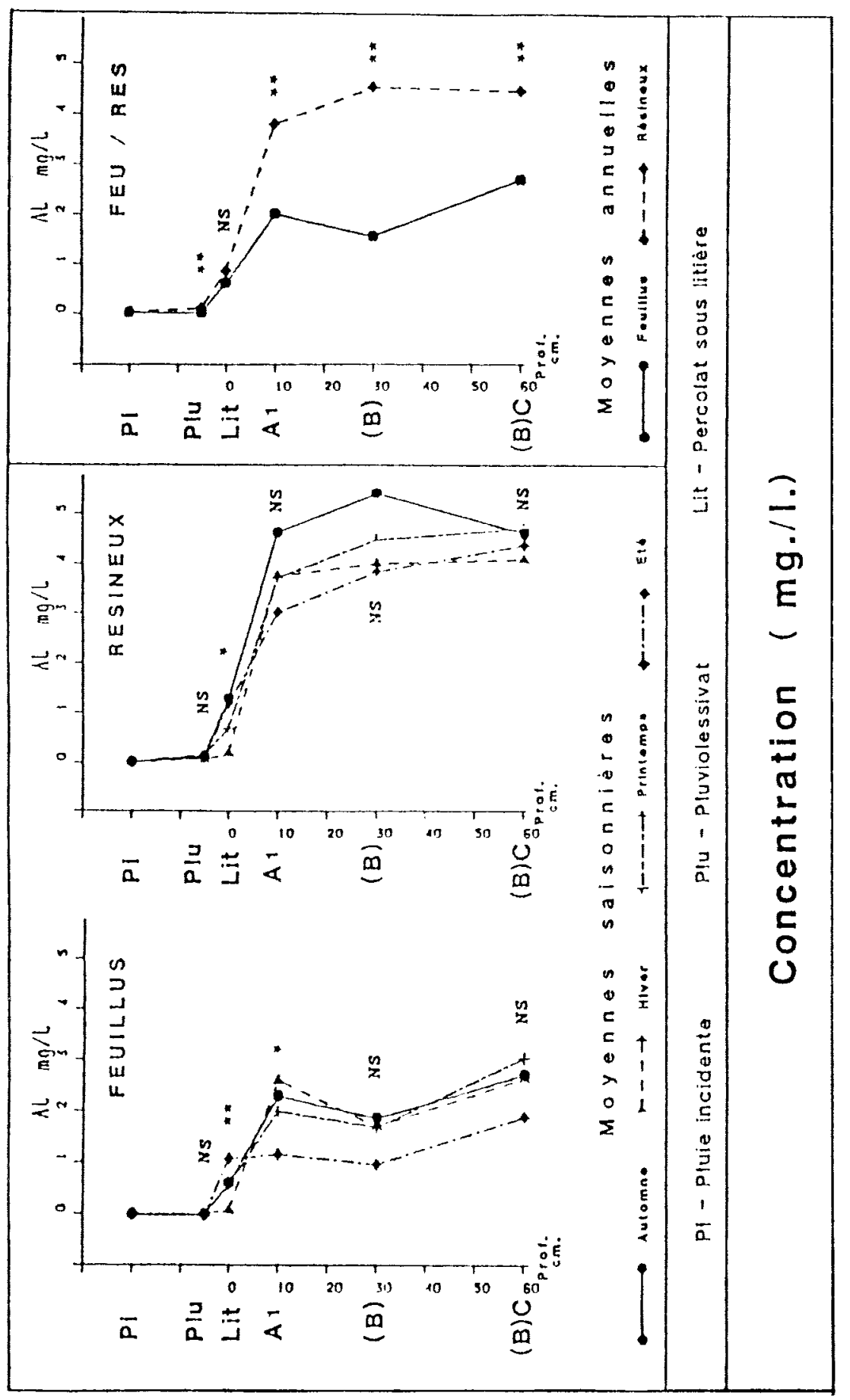




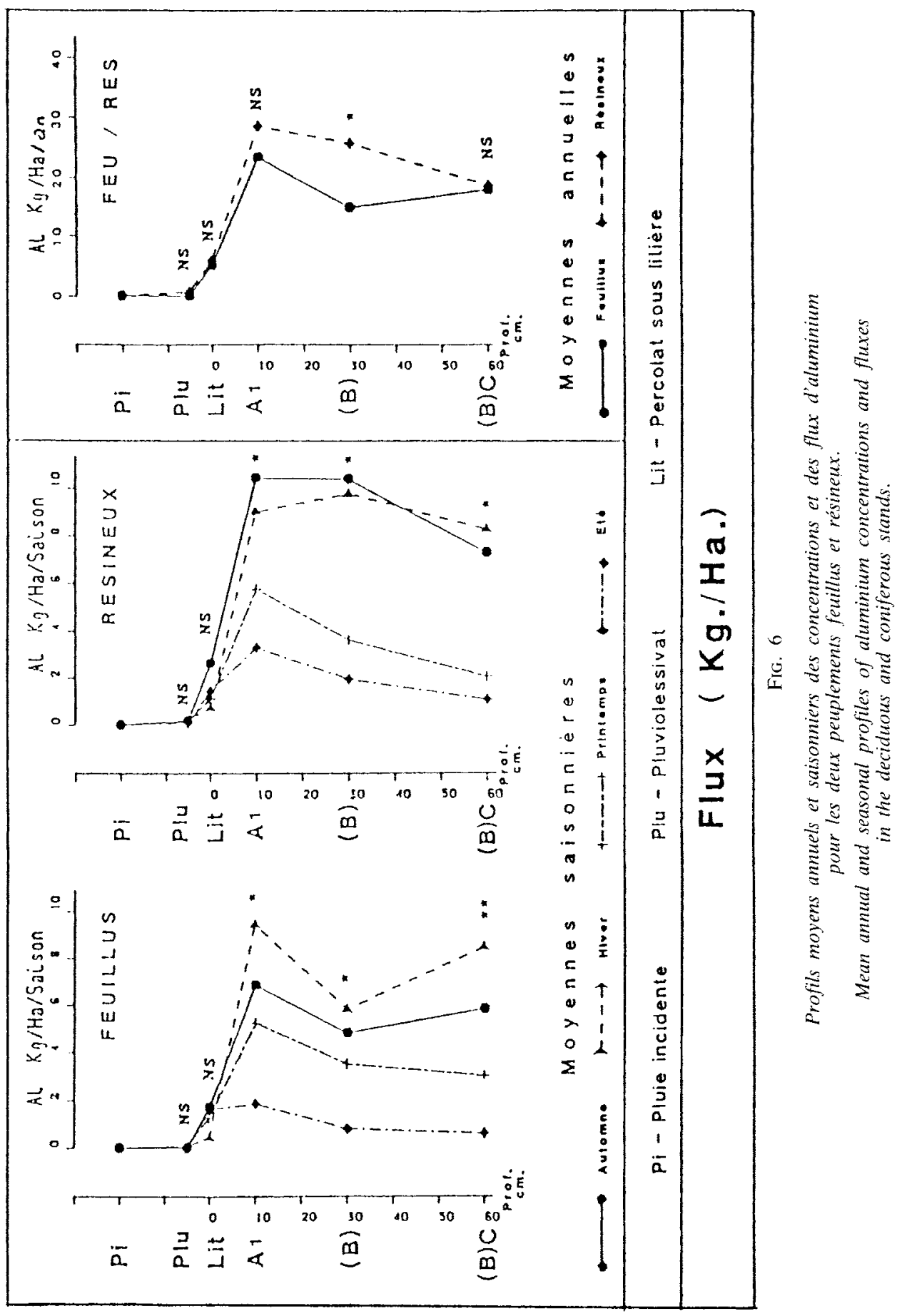


L'étude des caractéristiques du sol montre une évolution d'un sol brun acide sous un peuplement de feuillus vers un sol brun ocreux après 50 années de monoculture d'Epicéa commun.

Le lessivage des argiles constaté sous les épicéas (Nys, 1987) est, d'autre part, une source supplémentaire de perte d’aluminium sous forme de particules solides.

Ces modifications sont assez nettes pour que l'on tente de mesurer les modifications du fonctionnement de ces sols par la mesure des flux actuels.

\subsection{Flux de laluminium dans les eaux de gravité}

\subsection{Analyses élémentaires des solutions}

\section{- Evolution saisonnière}

La figure 5 permet de comparer pour les deux peuplements l'évolution saisonnière de l'aluminium par niveau. Cet élément est absent des pluies incidentes et des pluviolessivats sous les feuillus. Il est présent, à un niveau très faible, dans les pluviolessivats sous les résineux.

\section{-- Percolats des litières}

C'est le reflet de la minéralisation des litières. Les teneurs sont faibles et la variabilité dans le temps est importante sans liaison forte avec le cycle des saisons. Il n'y a pas de différence importante entre les deux espèces.

- Percolats des horizons du sol

La variation entre années est élevée, et, il semble que pour les épicéas, il y ait diminution progressive du niveau moyen des concentrations avec le temps. Ceci est l'inverse de la variation pour le calcium (Nys, 1987).

Est-ce dû à une minéralisation et libération de calcium sous les épicéas, liées à des changements microclimatiques à la suite de l'éclaircie accidentelle par tempête en 1979? En effet il n'y a pas de diminution nette sous le peuplement feuillu. Il y aurait eu ainsi un effet de traitement sylvicole (équivalent d'une éclaircie) qui se concrétise par une diminution de l'acidité et de son pouvoir agressif sur les minéraux. La figure 5 de l'évolution saisonnière de l'acidité, titration à $\mathrm{pH} 8,4$ confirme cette évolution. Elle montre en effet, au niveau des percolats dans le sol, un parallélisme avec celle de l'aluminium. Elle est l'inverse de celle du pH et du calcium (Nys, 1987). La différence liée à l'espèce semble diminuer avec le temps.

Ces évolutions de l'acidité dans le temps ne sont pas régulières d'une année à l'autre du fait de la variabilité climatique, et, probablement aussi, liées à des apports externes (soufre, azote, calcium)

\section{- Profils moyens des concentrations}

Le profil moyen des concentrations est l'image de la représentation du fonctionnement global de l'écosystème sol-végétation aux différents niveaux du peuplement et du sol. Sur la figure 6 sont représentés l'effet espèce (à droite) et, par espèce, l'effet saison (à gauche). 
Quasiment nulle dans les pluies incidentes et les pluviolessivats, la concentration en Al dans les eaux gravitaires augmente nettement sous les feuillus et les résineux au passage de l'horizon organo minéral $\mathrm{A}_{1}$. La constance des concentrations dans les horizons minéraux indique qu'il y a peu de libération dans ces horizons.

L'effet saison est faible par rapport à l'effet espèce. Cet effet espèce se caractérise

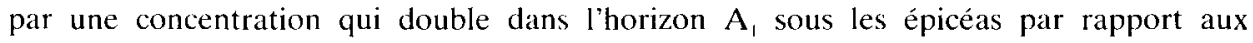
feuillus. L’aluminium est recyclé par la végétation mais à un niveau négligeable (dans le rapport $1 / 10^{6}$ ) en comparaison de celui du cycle géochimique dans les horizons du sol. C'est un des éléments caractéristiques du type de pédogénèse.

La différence des concentrations entre les espèces forestières peut avoir trois causes:

- soit un phénomène de dilution-concentration lié aux flux d'eau (mass-flow) différents entre les deux peuplements;

- soit un phénomène d'altération plus important comme le laissent à penser les résultats des caractéristiques des sols ;

- ou bien encore une conséquence secondaire d’apports exogènes différents.

Un calcul quantitatif par un bilan des flux a permis de trancher entre ces hypothèses.

\subsection{Le cycle de redistribution de l'aluminium par les eaux de gravité}

- Le cycle de l'eau

L'eau est le vecteur du transfert de l'aluminium et son flux a été mesuré au niveau du peuplement, le drainage dans le sol est estimé. L'effet saison est plus important que l'effet espèce et est lié au climat général de la station. L'effet espèce est dû essentiellement à l'interception par la cime des arbres. Sous feuillus, cette interception a lieu en période feuillée, ce qui représente environ 26 semaines pour le site des Ardennes.

La conséquence est une différence dans les sorties par drainage vertical profond (en $\mathrm{mm})$ :

\begin{tabular}{|c|c|c|c|c|c|}
\hline & Hiver & Printemps & Eté & Automne & Année \\
\hline Feuillus . . . . . . . & $322 \pm 68$ & $102 \pm 78$ & $25 \pm 30$ & $220 \pm 100$ & $669 \pm 100$ \\
\hline Résineux & $227 \pm 65$ & $42 \pm 45$ & $18 \pm 20$ & $162 \pm 83$ & $449 \pm 83$ \\
\hline$\% \quad \ldots$ & 29,5 & 58,8 & 28,0 & 26,4 & 32,9 \\
\hline
\end{tabular}

Ces différences de flux d'eau ne suffisent pas à expliquer, par le phénomène de mass-flow, les différences dans la composition des eaux de gravité mises en évidence entre les deux écosystèmes dans le paragraphe précédent ; en effet :

\section{- Profils moyens des flux d'aluminium}

Contrairement au fer (Nys, 1987) où l'apport se fait par la minéralisation des litières, l'aluminium se trouve en faible quantité dans la matière organique (biomasse, litière). Il participe peu, en valeur relative, au cycle biologique. 
TABIEAU 2

Matrice de corrélations $(\times 1000)$ entre flux d'éléments dans les eaux de gravité pour les différents niveaux dans l'écosystème.

Correlation matrix $(\times 1000)$ of element flux in gravitational water for the different levels in the ecosystems.

\begin{tabular}{|c|c|c|c|c|c|}
\hline & $\begin{array}{c}\mathrm{Al} \\
\mathrm{NO}_{3}\end{array}$ & $\underset{\mathrm{S}}{\mathrm{Al}}$ & $\begin{array}{l}\mathrm{Al} \\
\mathrm{C}\end{array}$ & $\begin{array}{c}\mathrm{Fe} \\
\mathrm{C}\end{array}$ & $\begin{array}{l}\mathrm{Fe} \\
\mathrm{Al}\end{array}$ \\
\hline Extéricur & NS ${ }^{0}$ & $\begin{array}{c}-241 \\
\text { NS }\end{array}$ & $\begin{array}{r}0 \\
\text { NS }\end{array}$ & $\begin{array}{c}-167 \\
N S\end{array}$ & $\begin{array}{r}0 \\
\text { NS }\end{array}$ \\
\hline
\end{tabular}

FEUILLUS

\begin{tabular}{|c|c|c|c|c|c|}
\hline Pluviomètre $\ldots \ldots \ldots$ & NS & NS ${ }^{0}$ & $\begin{array}{r}36 \\
\text { NS }\end{array}$ & $\begin{array}{c}-196 \\
\text { NS }\end{array}$ & $\begin{array}{r}8 \\
\text { NS }\end{array}$ \\
\hline Litière & $\mathrm{NS}^{82}$ & $\begin{array}{l}289 \\
\text { NS }\end{array}$ & $\begin{array}{l}208 \\
\text { NS }\end{array}$ & $\begin{array}{l}320 \\
\text { NS }\end{array}$ & 520 \\
\hline$A_{1}$ & $\begin{array}{l}272 \\
\text { NS }\end{array}$ & 637 & $\begin{array}{l}685 \\
* *\end{array}$ & $\begin{array}{c}-15 \\
\mathrm{NS}\end{array}$ & 516 \\
\hline$\ldots \ldots \ldots \ldots$ & $\begin{array}{l}700 \\
* * *\end{array}$ & $\begin{array}{c}707 \\
* *\end{array}$ & 541 & $\begin{array}{c}304 \\
\text { NS }\end{array}$ & 500 \\
\hline (B) $\mathrm{C}$ & $\begin{array}{l}797 \\
* * * *\end{array}$ & 678 & $\begin{array}{c}671 \\
* * *\end{array}$ & $\begin{array}{l}528 \\
*\end{array}$ & $\begin{array}{l}460) \\
\text { NS }\end{array}$ \\
\hline
\end{tabular}

RÉSINEUX

\begin{tabular}{|c|c|c|c|c|c|}
\hline Pluviomètre . . . . . . . & $\begin{array}{c}-299 \\
\text { NS }\end{array}$ & $\mathrm{NS}^{48}$ & $\begin{array}{l}219 \\
\text { NS }\end{array}$ & $\begin{array}{c}-103 \\
\text { NS }\end{array}$ & $\begin{array}{r}-32 \\
\mathrm{NS}\end{array}$ \\
\hline Litic̀re & $\begin{array}{l}327 \\
\text { NS }\end{array}$ & $\begin{array}{c}-454 \\
\text { NS }\end{array}$ & $\begin{array}{c}31 \\
\text { NS }\end{array}$ & $\begin{array}{l}381 \\
\text { NS }\end{array}$ & $\begin{array}{r}-15 \\
\mathrm{NS}\end{array}$ \\
\hline$A_{1} \ldots \ldots \ldots \ldots$ & $\begin{array}{c}828 \\
* * * *\end{array}$ & $\begin{array}{l}767 \\
* * *\end{array}$ & $\begin{array}{l}597 \\
* * *\end{array}$ & $\begin{array}{c}848 \\
* * * *\end{array}$ & $\begin{array}{l}430 \\
\text { NS }\end{array}$ \\
\hline (B) & $\begin{array}{c}841 \\
* * *\end{array}$ & $\begin{array}{c}587 \\
* * * *\end{array}$ & $\begin{array}{l}188 \\
\text { NS }\end{array}$ & $\mathrm{NS}^{71}$ & $\begin{array}{l}680 \\
* * *\end{array}$ \\
\hline (B) C . & $\begin{array}{l}910 \\
* * *\end{array}$ & $\begin{array}{l}737 \\
* *\end{array}$ & $\begin{array}{l}136 \\
\text { NS }\end{array}$ & $\mathrm{NS}^{28}$ & $\begin{array}{l}808 \\
* * *\end{array}$ \\
\hline
\end{tabular}

Le flux d'aluminium (fig. 6) augmente sous les deux peuplements, sous le $A_{1}$, lié à une libération ou à l'altération des minéraux du sol.

L'effet espèce était hautement significatif pour la concentration, mais pour le flux, la différence n'est significative qu'au niveau de l'horizon (B). Ceci s'explique par le phénomène du flux d'eau différent sous feuillus et résineux.

Les deux peuplements se différencient alors par un piégeage profond dans l'horizon (B) C sous les résineux. Les sorties par les eaux de drainage profond sous les horizons (B) C sont cependant équivalentes, $17,8 \mathrm{~kg} \cdot \mathrm{ha}^{-1} \cdot \mathrm{an}^{-1}$ sous les feuillus pour $18,5 \mathrm{~kg} \cdot \mathrm{ha}^{-1} \cdot \mathrm{an}^{-1}$ sous les épicéas. 


\section{- Liaisons spécifiques de l'aluminium}

Le tableau 2 des coefficients de corrélation entre flux d'éléments permet de constater l'existence, sous les résineux, de liaisons spécifiques entre éléments. Fer et Aluminium sont corrélés avec le Carbone sous l'horizon $\mathrm{A}_{1}$. Au niveau des horizons profonds du sol l'aluminium est hautement corrélé à l'azote nitrique et au soufre. Ces liaisons font penser à une migration conjointe de l'aluminium avec ces anions (NYS, Stevens, RanGer, 1986).

\section{Conclusion}

Un écosystème est considéré en équilibre, ou stable, lorsque les apports sont égaux aux sorties (Ulrich, 1982 ; VAN Miegroet, 1983 ; Waring, 1983 ; Ford et al., 1983).

Nous résumons par la figure 7 les données des cycles; le détail des mesures ou évaluation des valeurs des différents compartiments est donné par Nys (1987).

Les sorties exceptionnelles au moment des éclaircies ou coupes définitives représentent pour l'aluminium 0,3 et $0,5 \mathrm{~kg} \cdot \mathrm{ha}^{-1} \cdot$ an ${ }^{1}$ respectivement pour les feuillus et les résineux, soit des quantités très petites par rapport aux départs profonds pour les deux peuplements. Le bilan est un bilan de départ d'environ $18 \mathrm{~kg} \cdot \mathrm{ha}^{-1} \cdot$ an ${ }^{1}$ et cela pour les deux peuplements. L'effet espèce est faible dans le contexte d'une étude de bilan Entrée-Sortie de l'écosystème. Cet effet devient cependant significatif si l'on considère le fonctionnement à l'intérieur de ces écosystèmes.

Nous ne discuterons pas dans cet article des mécanismes différenciant l'immobilisation de l'aluminium dans les deux peuplements. Cela est discuté dans Nys et al. (1986) au sujet des relations de l'aluminium avec l'élément soufre.

L'aluminium provient pour une très faible part du cycle biologique ou du milieu extérieur. Il a pour origine essentielle l'altération au niveau de l'horizon $A_{1}$. Les analyses du fer et de l'aluminium libre ainsi que les indices $K_{\mathrm{Fe}}$ et $\mathrm{K}_{\mathrm{Al}}$ qui en sont déduits permettent de caractériser une évolution du complexe d'altération du sol lié à l'effet de l'espèce Epicéa commun. Ces valeurs caractérisent un état podzolisé plus avancé sous le résineux. Le sol brun acide à mull sous les feuillus a évolué vers un sol ocreux à moder sous les épicéas ; il y a donc enclenchement du processus de podzolisation sous les épicéas comme le confirme l'étude de fonctionnement des sols.

L'effet de l'introduction de l'Epicéa commun sur le fonctionnement d'un sol brun acide sous feuillus peut être résumé ainsi : il n'y a pas de différence due à l'espèce pour la récrétion: libération d'éléments et l'interception au niveau de la cime. Par contre le piégeage : adsorption sur le sol ou absorption par le végétal est plus ou moins rapide dans les horizons du sol pour les cations basiques et localisé essentiellement au niveau de l'horizon $A_{1}$.

L'effet espèce peut être défini comme un fonctionnement de type voisin pour les deux écosystèmes feuillus et résineux mais avec une intensité de ce fonctionnement différente caractérisée par une minéralisation plus lente sous les épicéas. Dans le sol l'altération différencie les peuplements car plus d'aluminium et de fer sont mis en solution sous le résineux dans l'horizon $A_{1}$ et la redistribution est plus profonde en (B) 
206

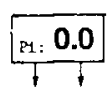

Aluminium

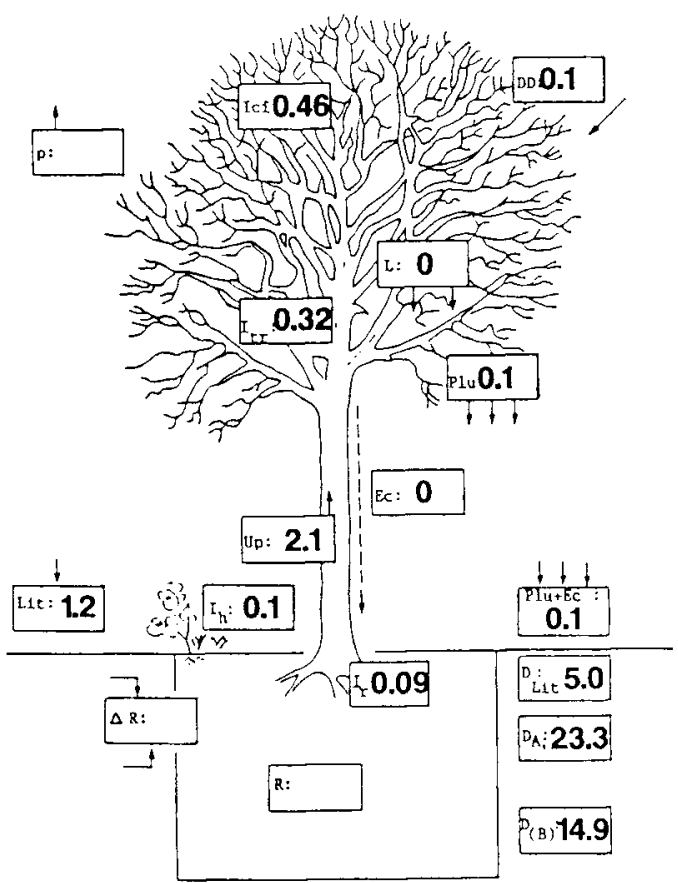

Feuillus
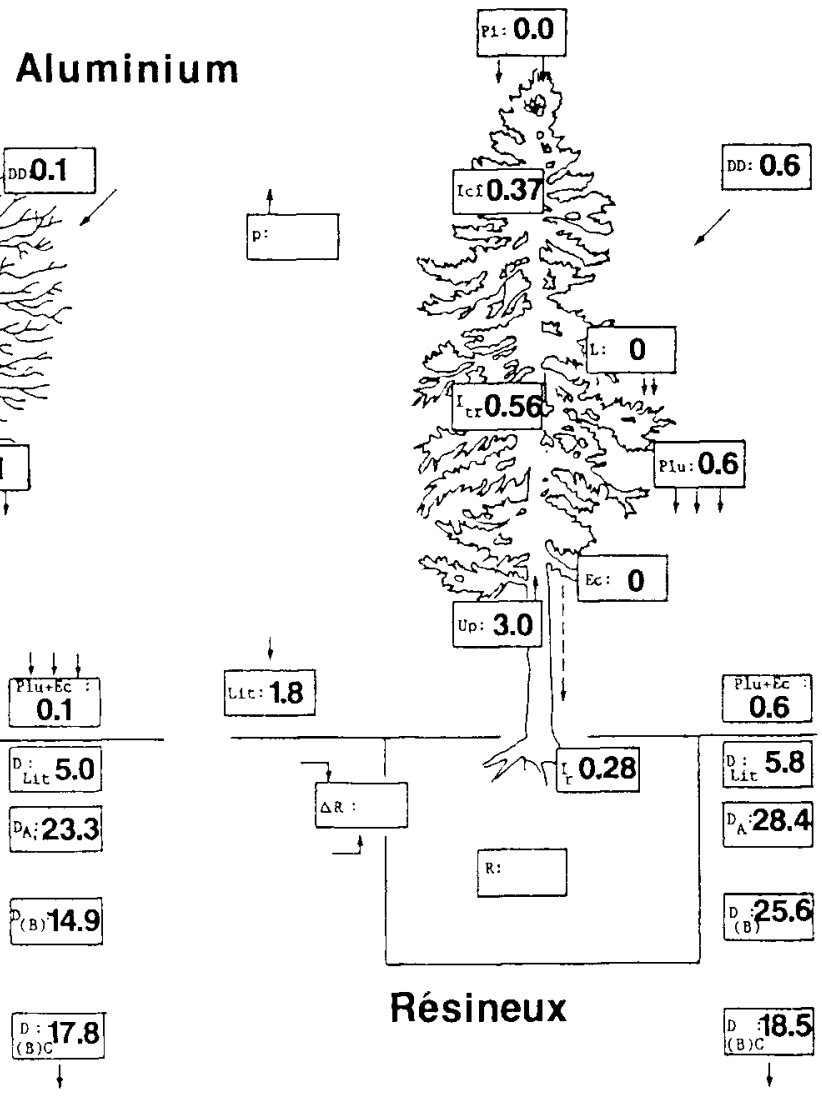

\section{Résineux}

FIG. 7

Immobilisation dans la biomasse et flux de l'élément aluminium dans les deux écosystèmes feuillus et résineux.

Biomass immobilisation and aluminium element flux in the deciduous and coniferous ecosystems.

- Pluies incidentes

- Dépôt sec

- Pertes par évaporation

- Retour par le cycle biologique

- Pluviolessivat

- Ecoulement le long du tronc

- Retour par litières + arbres morts

Transfert liquide dans le sol:

- sous litières

- sous $A_{1}(-10 \mathrm{~cm})$

- sous (B) $(-30 \mathrm{~cm})$

$-\operatorname{sous}(\mathrm{B}) \mathrm{C}(-60 \mathrm{~cm})$

- flux à l'émissaire

- Entrées latérales

- Sorties latérales

- Réscrves assimilables du sol

- Variations des réserves

- Prélèvement courant

Immobilisation dans :

- le tronc

- la cîme

- les racines

- la strate herbacée
$P_{i}$

DD

L

Plu

Ec

Lit

$\mathrm{D}_{\mathrm{l,t}}$

$\mathrm{D}_{\mathrm{Ai}}$

$D_{(B)}$

$\mathrm{D}_{\mathrm{SF}}$

$\mathrm{E}_{\text {l:at }}$

$\mathrm{D}_{\text {litt }}$

R

Up

$I_{1}$

$I_{\text {ti }}^{I_{\text {ti }}}$
Bulk precipitation

Dry deposit

Evaporation loss

Leaching

Throughfall

Stemflow

Litter + dead trees

Drainage :

- under litter

- under $A_{1}$ horizon

- under (B) horizon

- under (B) C horizon

- streamflow

- Lateral input

- Lateral output

- Available soil nutrients

- Variation of available soil nutrient

- Uptake

Immobilization in :

- the trunk

- the canopy

- the roots

- the herbaceous layer 
pour le fer et (B)C pour l'aluminium. Il n'y a donc pas de pertes supplémentaires hors du sol des éléments, mais redistribution à un niveau inférieur dans le sol.

La dynamique du flux de l'aluminium sous les épicéas semble être caractérisé par un double phénomène. Le premier, identique dans les deux peuplements, correspond à un entraînement d'une partie de l'aluminium libéré au niveau de l'horizon $A_{1}$ sous formes ioniques liées aux anions sulfates et nitrates. Le second décrit par Nys (1987) serait une redistribution, liée au carbone, du fer et d'une partie de l'aluminium, cela uniquement dans le sol sous les épicéas, laissant ainsi supposer une migration sous formes complexées de ces éléments. Ceci est en accord avec les résultats des analyses des formes libres dans le sol. Il y a donc différentiation faible mais significative du fonctionnement du sol sous l'effet du résineux.

La potentialité de la station est cependant maintenue pour ce qui concerne les cations basiques, éléments de la fertilité. Il y a peu de risque de pertes de production, sur ce type de station des Ardennes, à court ou moyen terme.

Reçu le $1^{\mathrm{kr}}$ décembre 1987.

Accepté le 24 février 1988.

\section{Références bibliographiques}

Aussenac G. 1975. Couverts forestiers et facteurs du climat: leurs interactions. conséquences ćcophysiologiques chez quelques résincux. Thèse Université Nancy $I$.

Delecour F.. Weissen F. Nanson A., 1967. Aspects techniques de l'échantillonnage des retombées annuelles et des horizons holorganiques des sols de forêts. Bull. Rech. Agron. Gembloux, tome II, n" 3, 429-449.

D.G.R.S.T., 1977. Modification de la fertilité des sols sous boisements artificiels de résineux purs. Publication INRA/C.R.F. B.P. 35, Seichamps.

Espiau P.. Pedro G., 1980. Caractérisation du complexe d'échange des sols acides. Le taux d'acidité d'échange et sa signification pédogénétique sous climat tempéré. Ann. Agron., 1980. 31 (4), 363-383.

Ford E., Milne R., Deans J., 1983. The response of trees to environmental fluctuations. In : State and change of forest ecosystems indicators in current research. Ed. Agren G., rcport 13 , Swedish University of Agricultural Sciences. Uppsala, 1984, 97-108.

Mlleter H.G.. 1984. Deposition plant soil interactions. Phil. Trans. R. Sc. Acad., B 305, 339-352.

Noirfalise A.. Vanese R., 1975. Conséquence de la monoculture des conifères pour la conservation des sols et pour le bilan hydrologique. Association des Espaces verts. Bruxclles.

Nys C., 1981. Modification des caractéristiques physico-chimiques d'un sol brun acide des Ardennes primaires par la monoculture d'épicéa commun. Ann. Sci. Forest., 38 (2), 237-258.

Nys C., 1987. Fonctionnement du sol d'un écosystème forestier : étude des modifications dues à la substitution d'une plantation d'épicéas commun (Picea abies) à une forêt feuilluc mélangée des Ardennes. Thèse Université Nancy $I$.

Nys C., RANGer J., 1981-1981-1983. Etude comparative de deux écosystèmes forestiers feuillus et résineux des Ardennes primaires françaises. I. Biomasse aérienne du taillis sous futaic. II. Biomasse aérienne d’une plantation équienne d'Epicéa commun (Picea abies Karst). III. Minéralomasse et cycle biologique. Ann. Sci. For., 38 (2), 259-282. Ann. Sci. For., 38 (3), 377-388. Ann. Sci. For, $40(1), 41-66$. 
Nys C., Stevens P., Ranger J., 1986. Sulphur nutrition of forests examined using a sulphur budget approach. In "Field methods in terrestrial nutrient cycling". Institute of Terrestrial Ecology. Merlewood, décembre 1986.

Souchifr B., 1971. Evolution des sols sur roches cristallines à l'étage montagnard (Vosges). Thèse d'Etat, Université de Nancy $I$.

Ulkici B., 1982. A concept of forest ecosystem stability and of acid deposition as driving force for destabilization. In : Effects of accumulation of air pollutants in forest ecosystems. Ed. Ulrich B. and Pankrath I., D. Reidel Publishing Company, Dordrecht, Holland.

VAN Miegroet M., 1983. Concepts of forest stability and forest management. In « State and change of forest ecosystems. Indicators in current research ». Ed. Agren G., report 13, Swedish University of Agricultural Sciences, Uppsala, 1984, 21-40.

WARING R., 1983. Unbalances ecosystems assessments and consequences. In "State and change of forest ecosystems. Indicators in current research". Ed. Agren G., report 13, Swedish University of Agricultural Sciences, Uppsala, 1984, 49-78. 